\title{
Ultraviolet irradiation improves the hydrophilicity and osteo-conduction of hydroxyapatite
}

Sho Kaneko, Yuji Yamamoto* (D), Kanichiro Wada, Gentaro Kumagai, Yoshifumi Harada, Ryota Yamauchi and

Yasuyuki Ishibashi

\begin{abstract}
Background: Treating a titanium or titanium alloy implant with ultraviolet (UV) light is known to improve its associated cell growth and osseointegration. However, little is known about the effect of UV irradiation on hydroxyapatite (HA), which is also used frequently in orthopaedic and dental surgery. Here we examined the effect of UV irradiation on the hydrophilicity of $\mathrm{HA}$, and on its osteoconduction ability in rats.

Methods: HA implants of low and high porosity were treated with UV light, and photofunctionalisation was assessed by the contact angle of a water drop on the surface. HA implants were also inserted into rat femurs, and the rats were killed 2 or 4 weeks later. The bone volume and bone area ratio were calculated from microcomputed tomography and histological data.

Results: The contact angle of a water drop on HA implants of both porosities was significantly reduced after UV irradiation. In the rat femurs, there was no significant difference in the bone volume between the UV light-treated and control implants at 2 or 4 weeks. The bone area ratio for the UV light-treated versus control implants was significantly increased at 2 weeks, but there was no significant difference at 4 weeks.

Conclusions: The surface of UV-irradiated HA disks was hydrophilic, in contrast to that of non-irradiated HA disks. Photofunctionalisation accelerated the increase in the bone area ratio in the early healing stage. This technology can be applied to surgical cases requiring the early fusion of bone and HA.
\end{abstract}

Keywords: Ultraviolet, Hydroxyapatite, Osseointegration, Photofunctionalisation

\section{Background}

It was recently shown that irradiating implants with ultraviolet (UV) light improves their associated cell growth and bone binding ability (osseointegration) [1, 2]. Irradiating the surface of titanium (Ti) or titanium alloy (Ti6Al4V) with ultraviolet rays of a short wavelength improves the material's osseointegration capacity. Irradiation changes the surface structure of $\mathrm{Ti}$ such that radicals are excited and the hydrophilicity is increased. Yamauchi et al. reported that the bone-implant contact

\footnotetext{
* Correspondence: yuji1112@hirosaki-u.ac.jp

Department of Orthopaedic Surgery, Hirosaki University Graduate School of Medicine, 5 Zaifu-cho, Hirosaki, Aomori 036-8562, Japan
}

(BIC) ratio for both $\mathrm{Ti}$ and Ti6Al4V UV-treated implants significantly increased at 2 weeks [3]. UV irradiation was also found to have an antimicrobial effect in the early stage after implantation [4].

Hydroxyapatite [Ca10(PO4)6(OH)2] (HA) is commonly used in orthopaedic and dental surgery because of its osteoconductivity and good biocompatibility. HA is used frequently in orthopaedic surgery, for example, to fill in bone defects, or to coat artificial joints, which promotes early fixation. To treat cervical myelopathy, HA is used in spinous process-splitting laminoplasty. In this procedure, the spines are split sagittally, and trapezoid-shaped HA spacers are inserted between the 
two halves to maintain an enlarged spinal canal [5]. Although it was reported that porous HA was a better material for osteoinduction [6], cases were reported in which bone fusion is insufficient or in which complications like dislocation occur, which were related to HA's osteogenesis ability [7].

Previous reports showed that UV irradiation physicochemically alters the HA surface [8-10]. Hydroxyl radicals on the surface of irradiated HA can increase its wettability and hydrophilicity, similar to the effect of photofunctionalised Ti. In an in vitro study using HA/ poly-L-lactic acid (PLLA), UV treatment improved the surface hydrophilicity without changing the mechanical strength, and cell adhesion to UV-treated HA/PLLA was significantly improved [11]. Therefore, the cell-adhesion ability to HA may increase by UV treatment due to the change in the surface wettability and hydrophilicity, leading to the improved osteoconductive ability of HA.

Here we examined the effect of UV irradiation on the hydrophilicity of HA and on HA's osteoconduction ability in rats. We hypothesised that UV irradiation would increase the hydrophilicity of the HA surface layer as it does for $\mathrm{Ti}$. To date, there has been little research on the influence of UV irradiation on HA in live animals.

\section{Materials and methods}

We performed an in vitro study to demonstrate the effect of UV irradiation on the hydrophilicity of the HA surface and an in vivo study to demonstrate the effect of
UV irradiation on the osteoconductive ability of HA. In the in vivo study, HA implants were inserted into rat femurs, and radiological analyses using microcomputed tomography and histological analyses using undecalcified specimens were performed. The study protocol (ethical code number: M16018) was approved by the Animal Research Committee of Hirosaki University, and all experiments were performed in accordance with the Rules for Animal Experimentation of Hirosaki University.

\section{Hydrophilicity of the hydroxyapatite surface}

HA disks (diameter $15 \mathrm{~mm}$, height $3 \mathrm{~mm}, 0 \%$ and $55 \%$ porosity: HOYA Technosurgical Inc., Japan) were used to evaluate the hydrophilicity of the HA surface. Four disks of each porosity were treated with UV irradiation for $15 \mathrm{~min}$ using a photo device (TheraBeam Affinity; Ushio Inc., Japan) (Fig. 1). The light source mounted in the TheraBeam Affinity is a low-pressure mercury $(\mathrm{Hg})$ lamp, which emits 185-nm and 254-nm UV light. Four disks that did not undergo UV irradiation were used as a control.

To examine the change in hydrophilicity on the disk surface after UV irradiation, the wettability was evaluated by measuring the contact angle of a water drop [3]. For this test, $10 \mu \mathrm{L}$ of water was dropped onto the disk surface before UV irradiation, and at $0 \mathrm{~min}, 1 \mathrm{~h}, 3 \mathrm{~h}, 6 \mathrm{~h}$, 2 weeks, and 4 weeks after UV irradiation. Moving images were taken at a rate of 240 frames per second (fps) using a high-speed camera (Casio EXILIM EX-ZR 1000, a

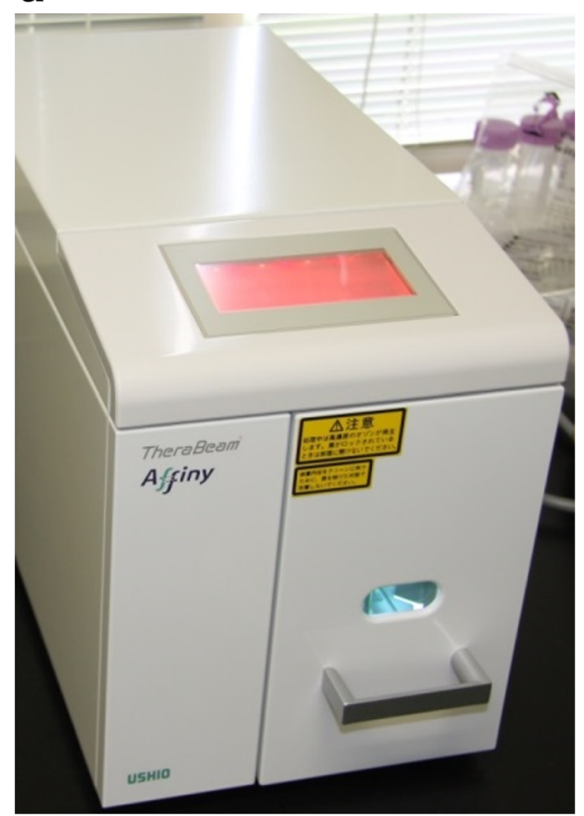

b

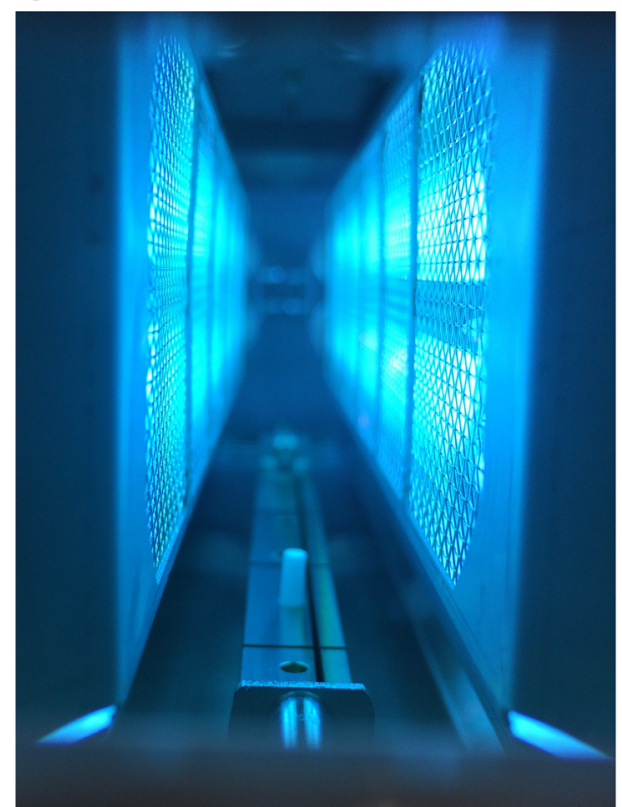

Fig. 1 Irradiation of HA implants with UV light. a UV photo device (TheraBeam ${ }^{\circledast}$ Affinity, Ushio Inc., Tokyo, Japan). b Implants were subjected to UV irradiation for $15 \mathrm{~min}$ 
Casio Computer Co., LTD., Japan). Using the still picture immediately after dropping, the angle of the water droplet with respect to the disk surface was measured by the $\theta / 2$ method [12]. After the water drop landed on the implant surface, the height (a) and contact diameter (b) of the drop were calculated by image analysis software

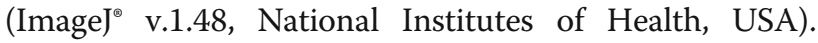
Using these measurements, the contact angle $(\theta)$ was calculated with the following formula: $\theta=2 \tan -1(2 \mathrm{a} /$ b). In this analysis, a low contact angle indicates surface hydrophilicity.

\section{Osteoconduction at hydroxyapatite}

Twenty HA cylinders (diameter $2.5 \mathrm{~mm}$, length $8 \mathrm{~mm}$, 55\% porosity: HOYA Technosurgical Inc., Japan) were used for the in vivo study. Half of the cylinders were treated with UV irradiation for $15 \mathrm{~min}$ as described above. The remaining 10 cylinders were used without UV treatment as a control.

Ten 8-week-old male Sprague-Dawley rats were used for the animal experiments. The rats were anaesthetised with 1 to $2 \%$ isoflurane. Both hind limbs were shaved, and the skin and fascia layers were opened separately. The flat aspect of each distal femur was exposed and used for implantation. The right and left distal femurs were drilled using a 3mm-diameter drill. UV-irradiated HA implants were inserted into the right femur holes, and HA implants without UV irradiation were inserted into the left ones. After implant placement, the skin and fascia were closed. Two or 4 weeks after the surgery, the rats were killed by intraperitoneal injection of pentobarbital, and the femurs were harvested. Five specimens were included in each group.

The specimens were fixed in 10\% buffered formalin and analysed using microcomputed tomography (Scan XmateL090, Comscantecno Co., Ltd., Japan). The imaging conditions were as follows: voltage, $80 \mathrm{kV}$; current, $100 \mu \mathrm{A}$; magnification, 4.942 times; resolution, $20.234 \mu \mathrm{m} /$ pixel; and slice thickness, $20.234 \mu \mathrm{m}$. Three-dimensional bone morphometric analysis was performed using the software (TRI/3D-BON, RATOC system engineering Co., Ltd., Japan). The mineralised bone volume (BV) ratio and tissue volume (TV) within $100 \mu \mathrm{m}$ from the implant surface were evaluated. The BV/TV ratio (also called the bone volume fraction), which is an important parameter for evaluating the microstructure of bone, was calculated as the bone volume (\%) in this area.

After microcomputed tomography, the specimens were embedded in methyl methacrylate without decalcification. The embedded specimens were then cut perpendicular to the long axis of the implant using a microtome. Each section was stained with VillanuevaGoldner to evaluate the bone area, which was stained green and observed by light microscopy (BZ-X700, Keyence Corp., Japan). To evaluate bone formation around the HA, the bone area (green) for each group was measured in ring-shaped regions $100 \mu \mathrm{m}$ outside and $100 \mu \mathrm{m}$ inside the HA surface by digital image analysis software (Image $\mathrm{J}^{\circ}$ v.1.48). The bone area ratio was calculated as the bone area divided by the total measured area (the HA area was subtracted), multiplied by 100 (\%).

\section{Statistical analysis}

Two-way analysis of variance with Tukey's post hoc test was performed to determine differences in the waterdrop contact angle. The Wilcoxon signed-rank test was performed to determine differences in the bone volume and bone area ratios between the UV(-) group and $\mathrm{UV}(+)$ group at 2 weeks or 4 weeks, respectively. The Mann-Whitney $U$ test was also performed to determine differences in the bone volume and bone area ratios at between 2 and 4 weeks within each group. Statistical analyses were performed using SPSS (v 22.0; IBM), and $p$ values $<0.05$ were considered significant.

\section{Results \\ Hydrophilicity of the HA surface \\ Contact angle analysis}

The water-drop contact angle indicated that both $0 \%$ porosity $\mathrm{HA}$ and $55 \%$ porosity HA had a hydrophobic surface before UV irradiation, with a mean contact angle of $53.9^{\circ}$ and $79.5^{\circ}$, respectively. After UV irradiation, both surfaces became hydrophilic, with contact angles of $17.7^{\circ}$ and $3.2^{\circ}$, respectively (Fig. 2). Over time, the contact angle on both surfaces increased, and the hydrophilicity decreased. In the $0 \%$ porosity HA group, there were significant differences between the $\mathrm{UV}(-)$ and $\mathrm{UV}(+)$ groups immediately, and at 1 and $3 \mathrm{~h}$ after irradiation. In the $55 \%$ porosity HA group, there were significant differences between the $\mathrm{UV}(-)$ and $\mathrm{UV}(+)$ groups immediately, and at 1, 3, 6, 12, and $24 \mathrm{~h}$ after irradiation (Fig. 3).

\section{Bone formation around HA samples Bone volume}

In the UV(-) group, the mean bone volume was $22.7 \pm$ $11.8 \%$ at 2 weeks and $28.9 \pm 9.7 \%$ at 4 weeks. In the $\mathrm{UV}(+)$ group, the mean bone volume was $30.2 \pm 10.1 \%$ at 2 weeks and $34.5 \pm 14.2 \%$ at 4 weeks. There was no significant difference between the $\mathrm{UV}(-)$ and $\mathrm{UV}(+)$ groups at 2 or 4 weeks $(p=0.080, p=0.345)$ (Figs. 4 and 5). An increase in bone volume was observed over time in each group; however, it was also not statistically significant difference $(p=0.421, p=0.754)$.

\section{Histological analysis}

In the UV(-) group, the mean bone area ratio was 14.1 $\pm 7.5 \%$ at 2 weeks and $34.8 \pm 8.6 \%$ at 4 weeks. In the 


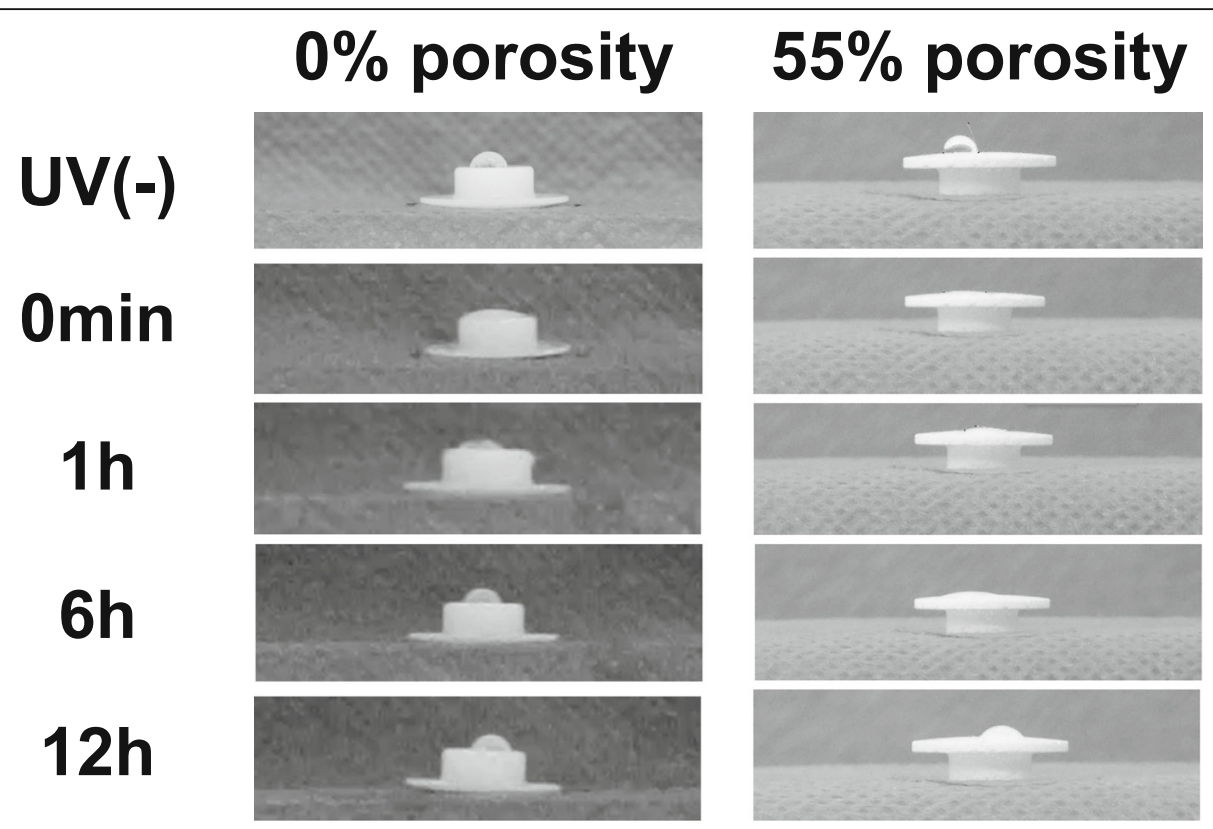

Fig. 2 Increased hydrophilicity of the HA surface after UV irradiation as evaluated by measurement of the water-drop contact angle. Photographs show a drop of water deposited onto $0 \%$ and $55 \%$ porosity HA disks

$\mathrm{UV}(+)$ group, the mean bone area ratio was $24.2 \pm 6.7 \%$ at 2 weeks and $36.3 \pm 17.4 \%$ at 4 weeks. There was a significant difference between $\mathrm{UV}(-)$ and $\mathrm{UV}(+)$ groups at 2 weeks $(p=0.043)$, but not at 4 weeks $(p=0.893)$ (Figs. 6 and 7). A statistically significant increase in bone area ratio was observed over time in the $\mathrm{UV}(-)$ group ( $p=$ $0.009)$; however, there was no statistically significant increase in the $\mathrm{UV}(+)$ group $(p=0.175)$.

\section{Discussion}

This study demonstrated that photofunctionalisation changed the HA surface from hydrophobic to hydrophilic regardless of the porosity and that the hydrophilicity was maintained for at least a few hours. The bone area in the early phase (at 2 weeks) after implantation was significantly higher in the $\mathrm{UV}(+)$ than in the $\mathrm{UV}(-)$ group.

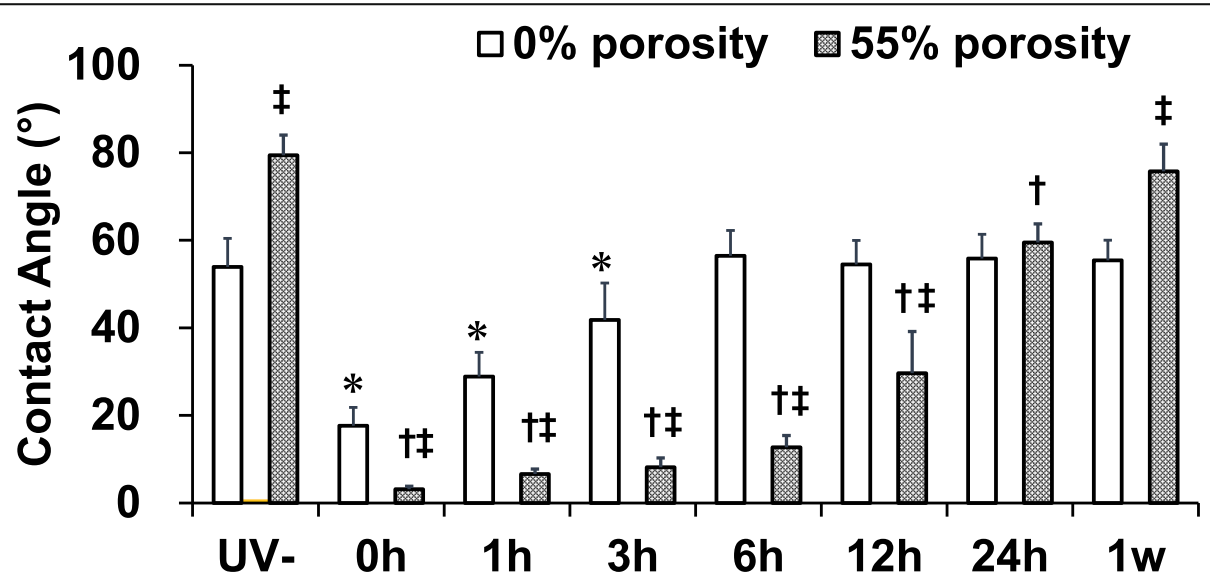

Fig. 3 The water-drop contact angle on the HA disk surface indicated hydrophobicity before UV irradiation and hydrophilicity after UV irradiation. The contact angle of both surfaces showed a time-dependent reduction in hydrophilicity. ${ }^{*} p<0.05,{ }^{*} 0 \%$ porosity UV(-) vs 0 min, $1 \mathrm{~h}$, and $3 \mathrm{~h}$ after UV irradiation; ${ }^{\dagger} p<0.05,{ }^{\dagger} 55 \%$ porosity UV(-) vs 0 min and 1, 3, 6, 12, and $24 \mathrm{~h}$ after UV irradiation; ${ }^{\ddagger} p<0.05,{ }^{\ddagger} 0 \%$ porosity vs $55 \%$ porosity at 0 min; $1,3,6$, and $12 \mathrm{~h}$; and 1 week after UV irradiation $(n=4)$ 

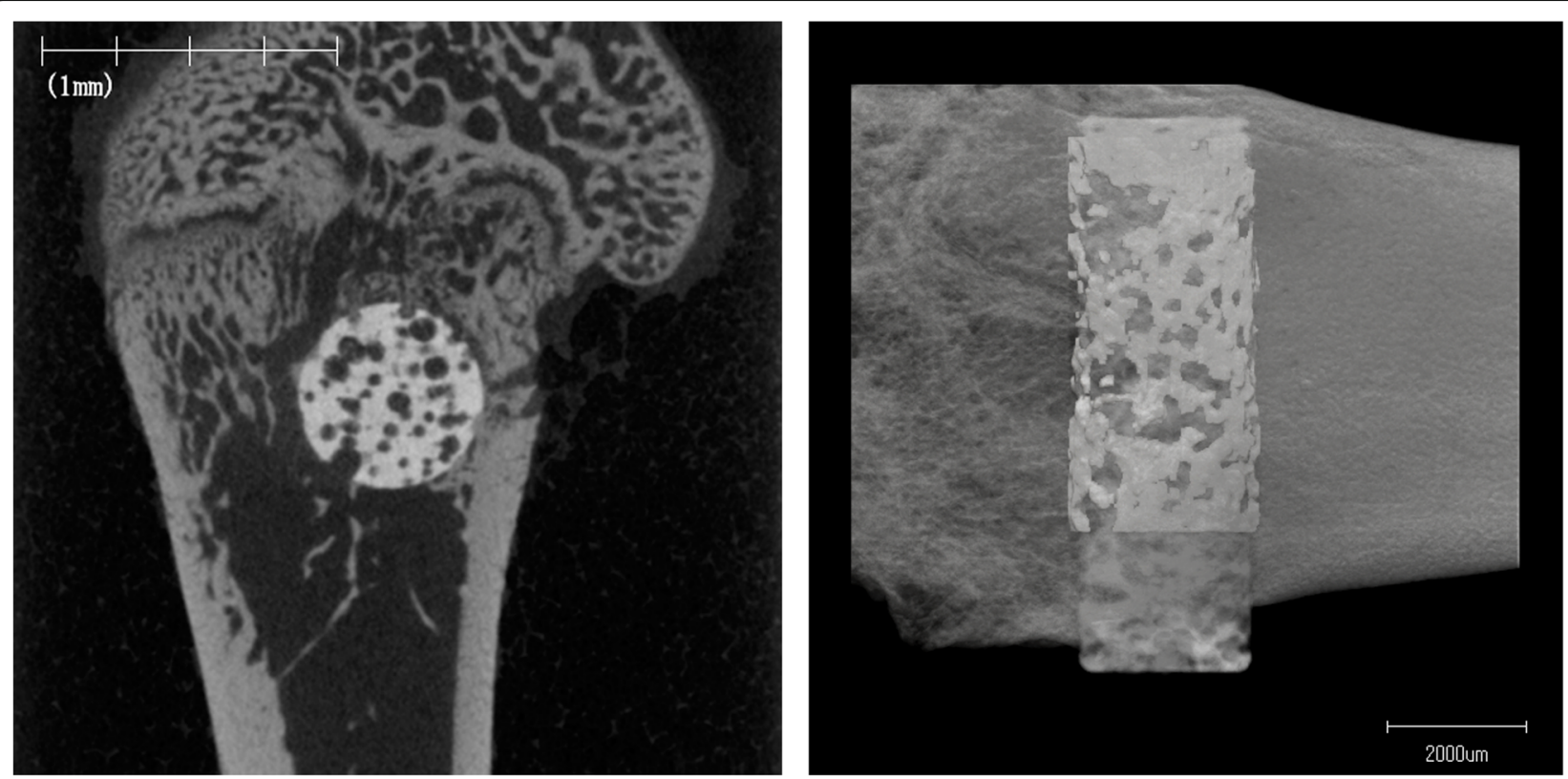

Fig. 4 Microcomputed tomography. The right image shows a representative cross-sectional micro CT slice in the perpendicular to the longitudinal axis of the implant. The left image shows a representative three-dimensional computed tomography image around the implant

$(\%)$

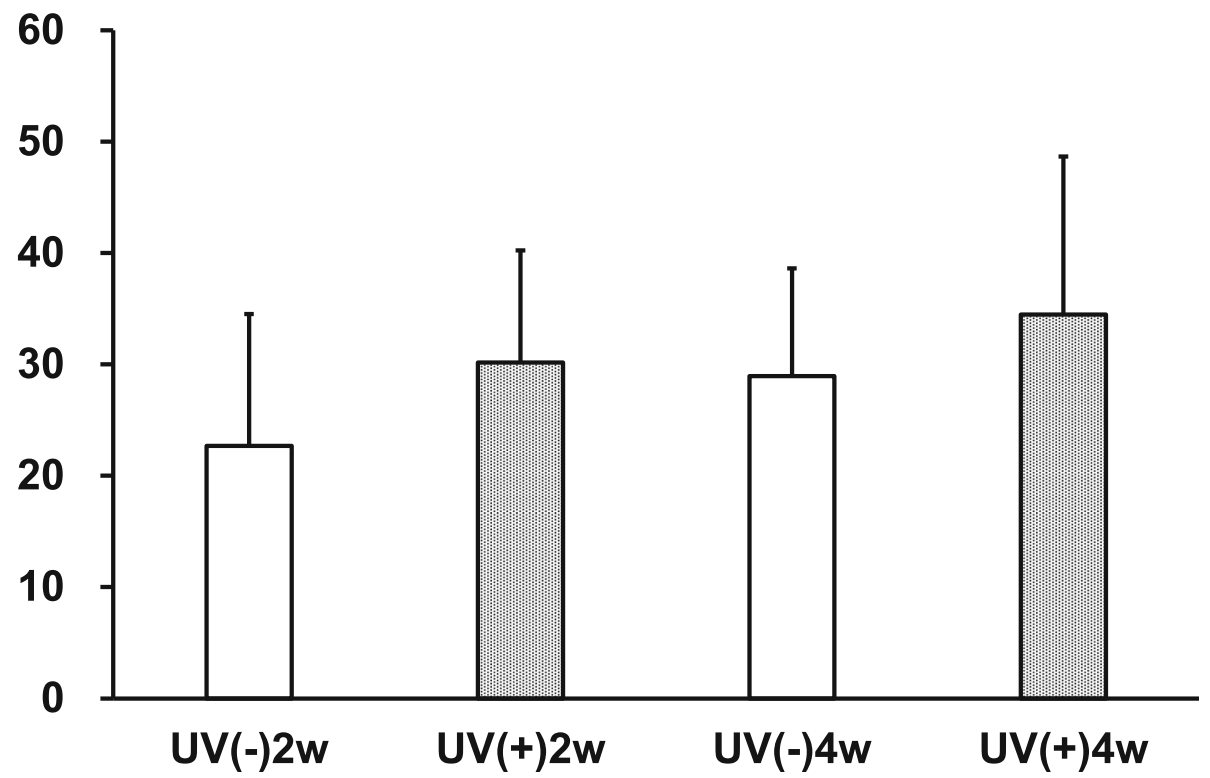

Fig. 5 The mean bone volume in the UV-irradiated HA group was higher than that of the non-irradiated group, but the difference was not statistically significant 

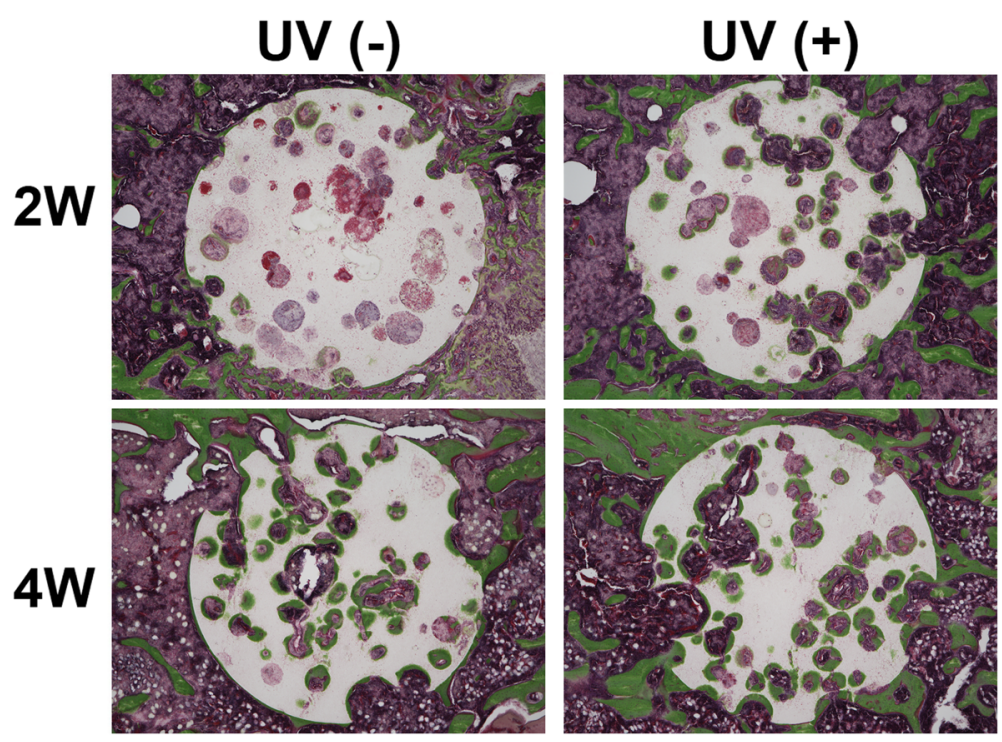

Fig. 6 Light microscopy images of Villanueva-Goldner staining 2 and 4 weeks after the implantation of HA into rats

There are several reports on the effect of photofunctionalised $\mathrm{Ti}$, which is a medical material. Photofunctionalised Ti implants are reported to increase the boneimplant contact ratio 2.5 times after 2 weeks, and 1.9 times after 4 weeks compared to Ti without photofunctionalisation [1]. In our previous study, Yamauchi et al. reported that UV-treated Ti and Ti6Al4V demonstrated significant differences in chemical properties and were more wettable than untreated implants [3]. Furthermore, we showed that antimicrobial activity was induced on $\mathrm{Ti}$ and Ti6Al4V for 7 days after UV irradiation [8]. UV irradiation removes carbon deposition from the Ti surface and exposes Ti4+ sites. It enhances the bioactivity of the surface by increasing its wettability and hydrophilicity.

\section{$(\%)$}

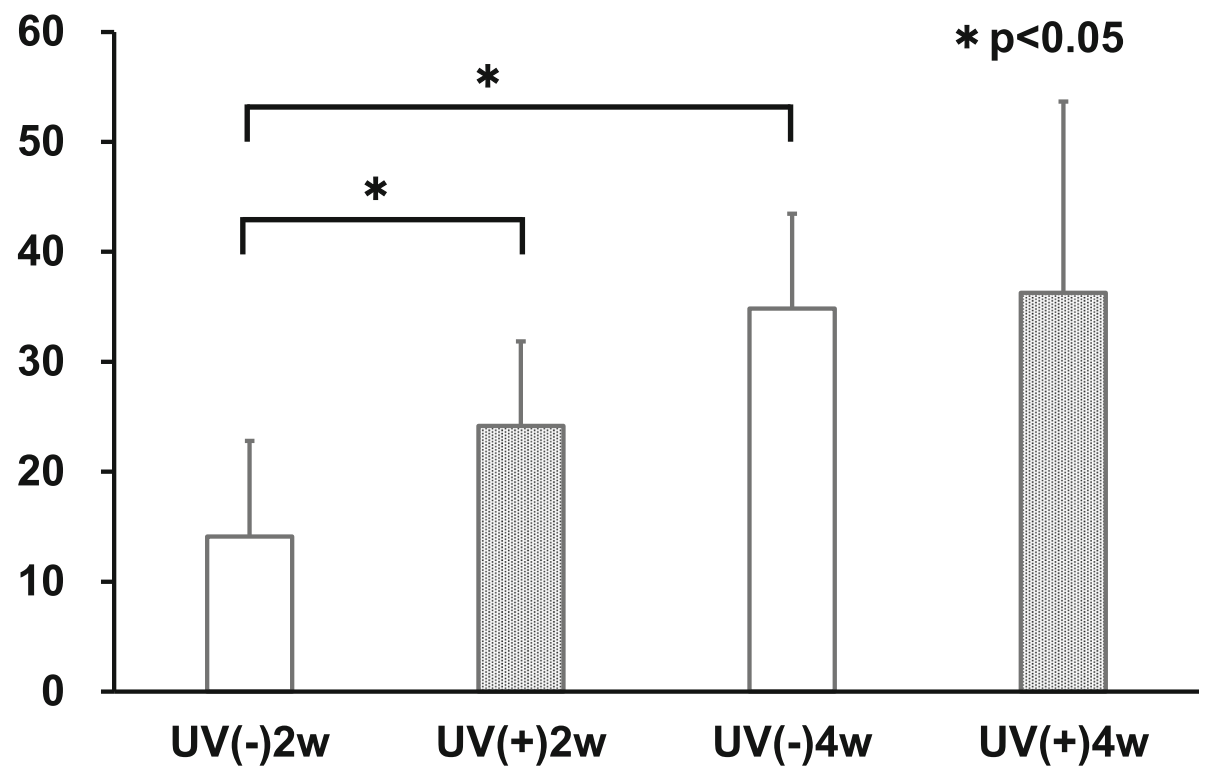

Fig. $\mathbf{7}$ The mean bone area ratio in the UV-irradiated HA group was statistically significantly higher than that of the non-irradiated group at 2 weeks; however, there was no significant difference at 4 weeks 
These changes enhance the initial attachment, proliferation, and differentiation of osteoblasts $[1,13]$.

This study demonstrated that the bone area around HA was greater in the UV-irradiated group than that in the control group at 2 weeks postoperatively. There are a few reports on the effects of ultraviolet irradiation on HA. Nishikawa reported that $\mathrm{O}_{2}^{-}$is generated by electron transfer to $\mathrm{O}_{2}$ after irradiating $\mathrm{HA}$ and that $\mathrm{OH}$ is produced by the reaction of $\mathrm{O}_{2}^{-}$and $\mathrm{H}_{2} \mathrm{O}$ [8]. It was proposed that the radical action in atmospheric air causes the decomposition of organic pollutants similar to the $\mathrm{TiO}_{2}$ photocatalyst [8]. Wakamura et al. investigated the effect of irradiating Ti-modified HA on the killing of colon bacillus [9]. They showed that $\mathrm{Ti}$ modified HA exhibits a higher bactericidal effect than $\mathrm{TiO}_{2}$ and that Ti-modified HA has both an absorption affinity for and a photocatalytic activity against microorganisms. They proposed that irradiation forms positive holes, which interact with absorbed $\mathrm{H}_{2} \mathrm{O}$ to yield hydroxyl radicals with a strong oxidation ability, which can decompose various organic materials as a bactericidal effect. Tanaka et al. analysed the decomposition of dimethyl sulphide on HA after UV irradiation using infrared spectroscopy and showed that the area intensity of $\mathrm{CH}$ bands due to dimethyl sulphide gradually decreases and that of surface $\mathrm{P}-\mathrm{OH}$ bands increases after irradiation [10]. These findings meant that surface P$\mathrm{OH}$ - radicals were formed, and dimethyl sulphides were decomposed by UV irradiation. Taken together, these previous reports showed that UV irradiation physicochemically alters the HA surface. Hydroxyl radicals on the surface of irradiated HA can increase its wettability and hydrophilicity, similar to the effect of photofunctionalised Ti. In an in vitro study using HA/PLLA, UV treatment improved the surface hydrophilicity without changing the mechanical strength, cell adhesion to UVtreated HA/PLLA was significantly improved, and cell differentiation was also significantly increased [11]. The cell-adhesion ability may increase due to the change in wettability and hydrophilicity, leading to the improved osteoconductive ability of HA in the early phase.

HA is widely used as a coating for uncemented total hip arthroplasty components. HA is an osteoconductive coating that has been shown to enhance implant fixation and accelerate bone growth [13, 14]. Autopsy retrievals showed the presence of extensive circumferential bone apposition on a HA-coated Ti femoral stem [15] and more bone ingrowth around femoral stems with HA coating than around those without HA [16]. The longterm follow-up of HA-coated stems has shown excellent clinical and radiographic outcomes [17-19]. A metaanalysis showed that HA-coated stems had better clinical scores and implant survival than porous-coated stems [20]. On the other hand, Schewelov et al. reported that some stem subsidence of fully HA-coated stems occurred [21]. In addition, in patients with a femoral neck fracture, 31 of 38 HA-coated stems migrated distally (mean value $2.7 \mathrm{~mm}$ ) during the first 3 months [22]. Our findings suggest that the UV irradiation of HA-coated stems might induce earlier biological fixation and prevent stem subsidence.

Iguchi et al. reported that high porosity HA-spaceraugmented laminoplasty produced good bonding-related results, as evaluated by computerised tomography [23]. However, they also reported that $4.4 \%$ of the spacers broke. Ono et al. reported two cases of dural damage from the dislocation of HA spacers due to absorption of the tip of the spinous process after cervical laminoplasty. Both patients underwent removal of the HA spacer and attained good neurological recovery [6]. Therefore, early union of the bone and HA spacer may enable early range of motion of the neck after cervical laminoplasty.

This study had some limitations. First, the biomechanical strength of the bone-HA integration was not tested. Second, the relationship between hydrophilicity and osteoconductivity after UV irradiation was not analysed. In addition, there are no data relative to cell adhesion or proliferation on HA after UV irradiation. Third, only five specimens were included in each group, making it difficult to come to a statistical conclusion. Post hoc power analysis indicated that five specimens provided a power of 0.6 to detect the difference of bone area between $\mathrm{UV}(-)$ and $\mathrm{UV}(+)$ groups (effect size $=1.3, \alpha=0.05$ ). Finally, the surfaces of the UV-irradiated and nonirradiated HA were not evaluated by electron microscopy. Further studies are needed to confirm the biomechanical strength of the UV-irradiated $\mathrm{HA}$ and the mechanism that promotes osseointegration in the early healing stage.

\section{Conclusion}

This study revealed that the surface of UV-irradiated HA disks were hydrophilic after UV irradiation, in contrast to the non-irradiated HA disks. Photofunctionalisation induced an accelerated increase in the bone area ratio in the early healing stage in rats. This technology could be applied to surgical cases requiring early fusion of the bone with HA.

\section{Abbreviations}

UV: Ultraviolet; HA: Hydroxyapatite; Ti: Titanium; Ti6Al4V: Titanium alloy; BIC: Bone-implant contact; BV: Bone volume; TV: Tissue volume

\section{Authors' contributions}

SK: acquisition of data, analysis and interpretation of data, and drafting the article. YY: study conception and design, data, analysis and interpretation of data, and drafting and revising the article. KW, GK, and $\mathrm{YH}$ : analysis and interpretation of data and drafting and revising the article. RY: analysis and interpretation of data and drafting and revising the article. YI: study conception and design, analysis and interpretation of data, and drafting and revising the article. All authors read and approved the final manuscript. 


\section{Funding}

There were no funding nor other sources of support to be disclosed. Neither the authors nor any members of their families have received any financial remuneration related to the subject of the article.

\section{Availability of data and materials}

The datasets used and analysed during the current study are available from the corresponding author on reasonable request.

\section{Ethics approval and consent to participate}

All animal procedures were performed under the approval and guidance of the Animal Research Committee of Hirosaki University (approval number: M16018).

\section{Consent for publication}

Not applicable.

\section{Competing interests}

The authors declare that they have no competing interests.

Received: 16 April 2020 Accepted: 7 September 2020

Published online: 18 September 2020

\section{References}

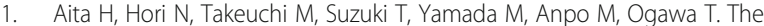
effect of ultraviolet functionalization of titanium on integration with bone. Biomaterials. 2009;30:1015-25.

2. Yadav A, Yadav R, Gupta A, Baranwal A, Bhatnagar A, Singh V. Effect of ultraviolet irradiation on the osseointegration of a titanium alloy with bone. Contemp Clin Dent. 2017:8:571-8.

3. Yamauchi R, Itabashi T, Wada K, Tanaka T, Kumagai G, Ishibashi Y. Photofunctionalised Ti6Al4V implants enhance early phase osseointegration. Bone Joint Res. 2017:6:331-6.

4. Itabashi T, Narita K, Ono A, Wada K, Tanaka T, Kumagai G, Yamauchi R, Nakane A, Ishibashi Y. Bactericidal and antimicrobial effects of pure titanium and titanium alloy treated with short-term, low-energy UV irradiation. Bone Joint Res. 2017;6:108-12.

5. Nakano K, Harata S, Suetsuna F, Araki T, Itoh J. Spinous process-splitting laminoplasty using hydroxyapatite spinous process spacer. Spine. 1992;17: S41-3.

6. Jang SJ, Kim SE, Han TS, Son JS, Kang SS, Choi SH. Bone regeneration of hydroxyapatite with granular form or porous scaffold in canine alveolar sockets. In Vivo. 2017:31:335-41.

7. Ono A, Yokoyama T, Numasawa T, Wada K, Toh S. Dural damage due to a loosened hydroxyapatite intraspinous spacer after spinous process-splitting laminoplasty. Report of two cases. J Neurosurg Spine. 2007;7:230-5.

8. Nishikawa H. Radical generation on hydroxyapatite by UV irradiation. Mater Lett. 2003:58:14-6.

9. Wakamura M, Hashimoto K, Watanabe T. Photocatalysis by calcium hydroxyapatite modified with $\mathrm{Ti}$ (IV): albumin decomposition and bactericidal effect. Langmuir. 2003;19:3428-31.

10. Tanaka H, Tsuda E, Nishikawa H, Fuji M. FTIR studies of adsorption and photocatalytic decomposition under UV irradiation of dimethyl sulfide on calcium hydroxyapatite. Adv Powder Technol. 2012;23:115-9.

11. Moroi A, Okuno M, Kobayashi G, Gamo H, Serizawa I, Yoshizawa K, Ikawa H, Ueki K. Effect on surface character and mechanical property of unsintered hydroxyapatite/poly-l-lactic acid (UHA/PLLA) material by UV treatment. J Biomed Mater Res B Appl Biomater. 2018;106(1):191-200.

12. Yang $M$, Lin S. A method for correcting the contact angle from the $\theta / 2$ method. Colloids Surf A Physicochem Eng Asp. 2003:220:199-210.

13. Sartoretto SC, Alves AT, Resende RF, Calasans-Maia J, Granjeiro JM, CalasansMaia MD. Early osseointegration driven by the surface chemistry and wettability of dental implants. J Appl Oral Sci. 2015;23:279-87.

14. Cook SD, Thomas KA, Kay JF, Jarcho M. Hydroxyapatite-coated titanium for orthopedic implant applications. Clin Orthop Relat Res. 1988;232:225-43.

15. Geesink RG. Osteoconductive coatings for total joint arthroplasty. Clin Orthop Relat Res. 2002;395:53-65.

16. Bauer TW, Geesink RC, Zimmerman R, McMahon JT. Hydroxyapatite-coated femoral stems. Histological analysis of components retrieved at autopsy. J Bone Joint Surg Am. 1991;73:1439-52.
17. Coathup MJ, Blunn GW, Flynn N, Williams C, Thomas NP. A comparison of bone remodelling around hydroxyapatite-coated, porous-coated and gritblasted hip replacements retrieved at post-mortem. J Bone Joint Surg (Br) 2001;83:118-23.

18. Shetty AA, Slack R, Tindall A, James KD, Rand C. Results of a hydroxyapatitecoated (Furlong) total hip replacement: a 13- to 15-year follow-up. J Bone Joint Surg (Br). 2005;87:1050-4.

19. Rajaratnam SS, Jack C, Tavakkolizadeh A, George MD, Fletcher RJ, Hankins M, Shepperd JA. Long-term results of a hydroxyapatite-coated femoral component in total hip replacement: a 15- to 21-year follow-up study. J Bone Joint Surg (Br). 2008:90:27-30.

20. Capello WN, D'Antonio JA, Geesink RG, Feinberg JR, Naughton M. Late remodeling around a proximally HA-coated tapered titanium femoral component. Clin Orthop Relat Res. 2009;467:155-65.

21. Chen YL, Lin T, Liu A, Shi MM, Hu B, Shi ZL, Yan SG. Does hydroxyapatite coating have no advantage over porous coating in primary total hip arthroplasty? A meta-analysis. J Orthop Surg Res. 2015;10:21.

22. Tv S, Ahlborg H, Sanzén L, Besjakov J, Carlsson A. Fixation of the fully hydroxyapatite-coated Corail stem implanted due to femoral neck fracture: 38 patients followed for 2 years with RSA and DEXA. Acta Orthop. 2012;83: 153-8.

23. Iguchi T, Kanemura A, Kurihara A, Kasahara K, Yoshiya S, Doita M, Nishida K. Cervical laminoplasty: evaluation of bone bonding of a high porosity hydroxyapatite spacer. J Neurosurg. 2003:98:137-42.

\section{Publisher's Note}

Springer Nature remains neutral with regard to jurisdictional claims in published maps and institutional affiliations.
Ready to submit your research? Choose BMC and benefit from:

- fast, convenient online submission

- thorough peer review by experienced researchers in your field

- rapid publication on acceptance

- support for research data, including large and complex data types

- gold Open Access which fosters wider collaboration and increased citations

- maximum visibility for your research: over $100 \mathrm{M}$ website views per year

At BMC, research is always in progress.

Learn more biomedcentral.com/submissions 